\title{
Amenazas de las manchas foliares de Sigatoka (Mycosphaerella spp.) en la producción
} sostenible de banano en el Ecuador

\section{Threats of leaf spots of Sigatoka (Mycosphaerella spp.) in sustainable ecuadorian banana production}

\author{
Jorge García Regalado ${ }^{1}$; Augusto Marcillo Plaza ${ }^{2}$; Christian Palacios Sánchez ${ }^{3}$
}

${ }^{1}$ Economista, Docente, Universidad Agraria del Ecuador. Contacto: igarcia@uagraria.edu.ec; ${ }^{2}$ Ingeniero Agrónomo, MSc. Docente, Universidad Agraria del Ecuador. Contacto: jmarcillo@uagraria.edu.ec; ${ }^{3}$ Técnico Especializado, Instituto de Investigaciones de Economía Agrícola y Desarrollo Rural, Universidad Agraria del Ecuador. Contacto: christianpalaciossanchez@ gmail.com

R E V I S Ã O

Recebido: 28/05/2019

Aprovado: 06/11/2019

Palabras clave:

Sigatoka-negra

Enfermedades foliares

Impacto económico

\section{Key words:}

Black sigatoka

Foliar diseases

Economic impact

\section{INTRODUCCIÓN}

El cultivo de banano en el Ecuador es importante en el ámbito comercial y social, representa el 2\% del PIB (Producto Interno Bruto) general y aproximadamente el 35\% del PIB agrícola, este cultivo genera empleo a 1 millón de familias y beneficia a 2,5 millones de personas aproximadamente, que representa el 6\% de la población total del país (MCE, 2017).

Durante este mismo año el Instituto Nacional de Estadística y Censos (2017) en la encuesta de superficie y producción agropecuaria continua, expone que la superficie nacional bananera presentó un decremento de 9,98\%, la superficie cosechada también presenta un decrecimiento del 12,35\% en comparación a los datos del año 2016.

$\mathrm{La}$ industria bananera es clave para el desarrollo económico agrícola por lo que la preocupación clave para la producción bananera son las consecuencias del aumento de incidencia de plagas y enfermedades provocadas por el clima, afectando en los rendimientos futuros del banano y su vialidad a largo plazo como lo expone la Organización de las Naciones Unidas para la Alimentación y la Agricultura FAO (2015) mediante su estudio sobre el cambio climático y sostenibilidad del banano en el Ecuador (ELBEHRI et al., 2015). Además, la FAO (1999) determina que las enfermedades son el principal factor limitante de los cultivos y que los países productores invierten considerables sumas de dinero en los estudios de investigación, transferencia y control de las mismas, en Ecuador como en la mayaría de países de Latinoamérica y el Caribe las enfermedades constituyen también el mayor obstáculo para la producción.

Actualmente las principales enfermedades foliares en el cultivo de Banano son las manchas foliares por Sigatoka denominas así por Jones (2000). Estas enfermedades son causadas por dos patógenos del mismo género (Mycosphaerella), el primero en aparecer fue la conocida como Sigatoka-amarilla cuyo agente causal es (Mycosphaerella

\section{Revista Verde}

ISSN 1981-8203

Pombal, Paraíba, Brasil v. 14, n.5, Edição Especial, p.591-596, 2019

doi: $10.18378 /$ rvads.v14i4.6623 
musicola Leach et Mulde), posteriormente con una mayor agresividad la mancha de la raya negra conocida comúnmente como Sigatoka-negra causada por el patógeno (Mycosphaerella fijensis Morelet).

Las manchas foliares de Sigatoka representan el principal problema fitopatológico y la mayor limitante para la producción sostenible de bananas a nivel mundial, que repercute en el precio de producción por los elevados costos que generan las contrataciones de los servicios de fumigaciones aéreas para poder realizar los controles de la enfermedad provocando, además, el aumento de frecuencia en las fumigaciones con fungicidas que produce un impacto negativo en el medio ambiente (GUZMAN et al., 2013).

El presente artículo describirá las mencionadas enfermedades que se han diseminado en la mayoría de los países productores del cultivo de Banano, analizará el caso en Ecuador y como estas enfermedades impactan en la evidente variación en la producción y la calidad del banano ecuatoriano.

\section{MATERIAL Y MÉTODOS}

La presente investigación se realizó mediante la revisión teórica de las incidencias de las enfermedades de manchas foliares de Sigatoka, posteriormente, se analizan los datos del sector bananero ecuatoriano desde fuentes oficiales e instituciones gubernamentales, esta investigación es de carácter cualitativo y busca evidenciar que existen factores fitopatológicos que tienen gran importancia en un sistema de producción bananera. Se utilizó información de agrupaciones bananeras nacionales y se comparó con casos y estudios en otros países como: Brasil, Honduras, Costa Rica, Cuba, Panamá y Colombia.

El levantamiento de información se fundamenta en artículos indexados, procurando que los mismos contengan numerosas citas relevantes en la investigación, que sean de origen científico. Siguiendo los pasos de búsqueda de literatura, exploración de la literatura, desarrollo del argumento y crítica de la literatura, la selección y organización de la información permite la elección de información con mayor potencial en la presente investigación.

\section{RESULTADOS Y DISCUSIÓN}

\section{Manchas foliares de Sigatoka}

El primer reporte de la enfermedad de manchas foliares por Sigatoka, específicamente de Sigatoka-amarilla, se presentó en la isla de Java por Zimmerman (1902) y consecutivamente en el valle de Sigatoka en la isla de Viti Levu, Fiji, de donde nace el característico nombre aun usado por los agricultores (MOURICHON; FULLERTON, 1992).

La Sigatoka-amarilla cuyo agente causal es Mycosphaerella musicola Leach (anamorfo Pseudocercospora musae [Zimm.] Deighton), fue la enfermedad foliar con mayor importancia en el banano donde se informó cuantiosas pérdidas en 1912 (JONES, 2000), se dispersó rápidamente en los años de 1930 y llevo a pensar que las esporas de los hongos eran esparcidas por las corrientes de aire, teoría que fue desestimada por Jones (2000) al aclarar que la eficiente dispersión por largas distancias solo puede producirse por el movimiento de material vegetal sin control sanitario.

La enfermedad de la raya negra conocida hoy mundialmente como Sigatoka-negra cuyo agente causal es Mycosphaerella fijensis Morelet (anamorfo Pseudocercospora fijensis [Morelet] Deighton), descrita inicialmente en la isla de Fiji (1963) en el valle de Sigatoka donde también fue reportada la Sigatoka-amarilla, aunque esta enfermedad debería llamarse raya negra, su nombre Sigatoka-negra ha sido ampliamente aceptado en todas las zonas productoras de banano (MARÍN et al., 2003). Podemos analizar el año de aparición de la enfermedad empezando por Fiji en 1963, Honduras en 1972, Colombia en 1981 y en Ecuador los primeros reportes datan en 1989 (MARÍN et al., 2003).

Las manchas foliares de Sigatoka presentan una sintomatología similar que consisten en manchas pardas al inicio, que se tornan agresivas hasta formar lesiones necróticas y halos amarillentos con el centro ligeramente gris, los daños causados por la Sigatoka-negra son más visibles que los causados por Sigatoka-amarilla substancialmente en el envés de la hoja y los daños principalmente en hojas jóvenes, por lo que produce un daño en el tejido foliar de la planta y se considera más peligrosa que la Sigatoka-amarilla (POLANCO et al., 2002).

Las lesiones pueden destruir áreas importantes de tejido foliar, lo cual reduce la capacidad natural de realizar fotosíntesis en la planta y disminuir la proporción de fotoasimilados disponibles para la planta, decayendo en su productividad, además de inducir a la madurez prematura de los frutos que impactará directamente con el rendimiento y producción de la plantación (FULLERTON; OLSEN, 1995; HIDALGO et al., 2006). Las enfermedades foliares por Sigatoka. afectan mundialmente a la producción bananera, causando diversas problemáticas como apreciamos en la Tabla 2.

Tabla 2: Problemáticas de las manchas foliares de Sigatoka por país.

\begin{tabular}{clll}
\hline Casos & \multicolumn{1}{c}{ Problemática } & \multicolumn{1}{c}{ Origina } & Fuente \\
\hline Costa Rica & $\begin{array}{l}\text { La Sigatoka-negra puede representar un costo } \\
\text { adicional del 25\% del costo total de } \\
\text { producción. }\end{array}$ & $\begin{array}{l}\text { Costos elevados en tratamientos } \\
\text { para la Sigatoka-negra. }\end{array}$ & (GAUHL, 1994) \\
\hline \multirow{2}{*}{ Honduras } & $\begin{array}{l}\text { En el año de 1976 sufre una caida del 85\% de } \\
\text { la cantidad de cajas exportadas de plátano a } \\
\text { causa de la Sigatoka Negra. }\end{array}$ & $\begin{array}{l}\text { Reducción de las exportaciones } \\
\text { de banano. }\end{array}$ & (MARÍN; ROMERO, 1998) \\
\hline \multirow{2}{*}{ Cuba } & $\begin{array}{l}\text { La fumigación preventiva llegó a alcanzar los } \\
\text { 23 ciclos y los costos de protección alcanzarón } \\
\text { el 15\% del costo total de producción. }\end{array}$ & $\begin{array}{l}\text { Aumento de fumigaciones al } \\
\text { año, aumento del costo de } \\
\text { protección, impacto ambiental } \\
\text { negativo. }\end{array}$ & (PÉREZ et al., 1993) \\
\hline
\end{tabular}


La industria bananera se ve cada vez mas

Ecuador afectada por los elevados costos de producción que representa el control de Sigatoka-negra.

Brazil reducen la vida verde considerablemente del banano en la post cosecha.
Industria Bananera afectada por

controles dificiles de costear.

(FAO, 2005)

Maduración precoz,

complicaciones en la logistica y ～(GUZMAN et al., 2013)

transporte
La Sigatoka-negra, ha desplazado a la Sigatoka-amarilla debido a su mayor tolerancia a la variación de la temperatura, ambas especies pueden coexistir (GUZMAN et al., 2013). La Sigatoka-negra dio su primer gran golpe en plantaciones de Honduras a mediados de los años setenta. La Sigatoka-negra es una de las enfermedades más interesantes que se estudian en las regiones productoras de banano, es la mayor amenaza en el último siglo (GUZMÁN, 2003).

La variación de clima en el Ecuador ha permitido que las enfermedades foliares por Sigatoka tengan un comportamiento diverso en las distintas provincias productoras de banano en el país, después de la presencia del fenómeno del niño en 1998 la Sigatoka-negra aumento su agresividad (porcentaje de daño foliar y diseminación de las esporas del hongo) por ello muchos programas de control de la enfermedad fracasaron. Las bananeras perdieron mucha fruta destinada a la exportación, con consecuencias económicas graves para los productores y exportadores de banano como la pérdida de capital para continuar con los procesos de producción (MARTILLO; SOLANO, 2003). Como consecuencia se registró una disminución de la superficie plantada en un $31,75 \%$ de banano a nivel local como se muestra en la Figura 1.

Figura 1. Superficie Plantada en el Ecuador entre los años 2002 y 2018

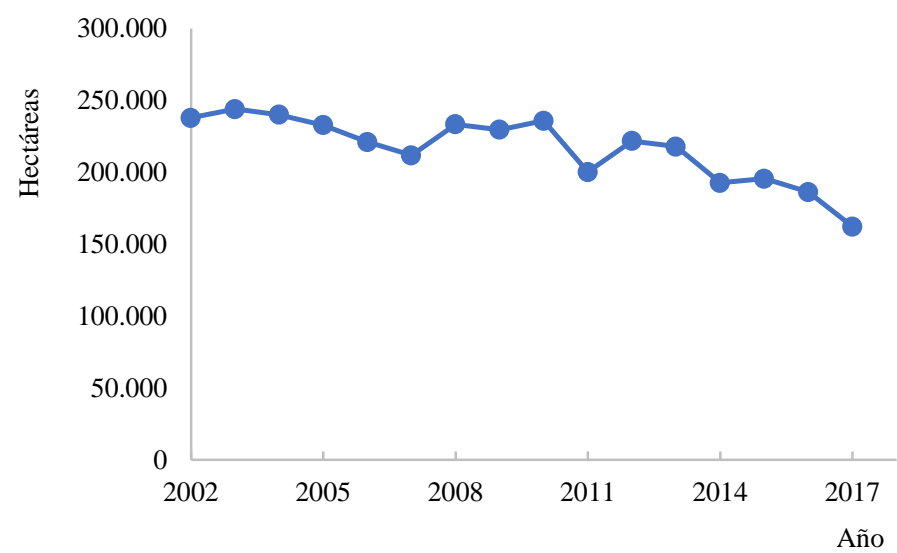

Fuente: (INEC, 2017)

\section{Perjuicios económicos de la Sigatoka Negra}

La Sigatoka-negra, desplazando a la Sigatoka-amarilla y actualmente produciendo las mayores repercusiones económicas en plantaciones comerciales de América Central, Panamá, Colombia y Ecuador, así como afecta a algunas regiones de Asia y África (MARÍN et al., 2003).

Existen factores que están estrechamente relacionado con el desarrollo de la enfermedad y la aparición de sus síntomas como: altas temperaturas, humedad relativa alta, elevada cantidad de precipitación, velocidad del viento y otras condiciones relacionadas a las labores culturales y labores mal desarrolladas dentro de las plantaciones bananeras como lo indica Orozco et al., (2008) cuyos factores contribuyen a la propagación y diseminación de la enfermedad que afecta al control y las pérdidas son inevitables para la mayoría de productores (GUZMAN et al., 2013).

Entre los factores que preocupan de forma especial a los productores bananeros ecuatorianos según el informe de Coyontura presentado por el BCE (2015) son los altos costos de los insumos y los factores climáticos adversos para la producción, como resultado de estos factores tenemos una alta incidencia de las manchas foliares por Sigatoka y las numerosas aplicaciones anuales de fungicidas que afecta directamnte la sostenibilidad de producción bananera convencional.

Los programas actuales para el control de Sigatoka-negra presentan elevados costos por que se basan en la aplicación de fungicidas en muchas ocasiones por vía aérea, en el Figura 2 podemos observar la superficie nacional que cuenta con 162.234 Ha de banano, entre ellas se representan las que se encuentra bajo fumigación aérea correspondiente a la producción de banano en el año 2014. Los ciclos de repeticiones de estas aplicaciones se han incrementado con dosis más elevadas de productos fungicidas. Las aplicaciones deben repetirse año tras año de manera indefinida y cada año con costos aún mayores (SOTOVER, 1972; MARÍN et al., 2003).

Figura 2. Superficie ecuatoriana bajo fumigación aérea de fungicidas en el año 2014



- Superficie Plantada - Superficie bajo fumigación aerea

Fuente: Catastro bananero (2014)

En Ecuador el banano es un producto de gran importancia económica, como lo podemos apreciar en el Figura 3. esta industria se ve fuertemente afectada por los elevados costos de producción, entre ellos se incluyen los que incurren en el 
control de la enfermedad de manchas foliares por Sigatoka que representa hasta un $30 \%$ aproximadamente del costo total de producción (FAO, 2005).

Figura 3. Representación nacional del control de Sigatokanegra en el costo total de producción en Ecuador.

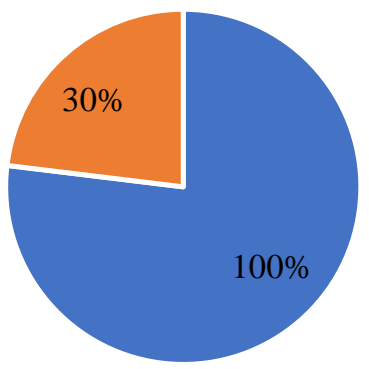

\section{- Otros Costos $\quad$ Control de Sigatoka}

Las grandes bananeras pueden asumir costos de fumigación de forma aérea y repetidos ciclos, pero los pequeños productores no pueden asimilar los altos precios de estos controles químicos, volviendose vulnerables ante la enfermedad y sufir cuantiosas perdidas económicas por lo que representa un problema no solo de origen económico sino tambien social, ya que la redución de pequeños productores reduce la superficie cosechada cada año e influye en una baja tasa de rendimiento que oscila entre 1700 y 2000 cajas/hectárea/año en el banano de exportación. (MARÍN; ROMERO, 1998; MCE, 2017). Podemos observar como ejemplo en la Figura 4. el caso de la hacienda Clementina ubicada en la provincia de Los ríos canton Babahoyo que aumentó en un 36,59\% el número de aplicaciones de fungicidas por medio de fumigaciónes aérea en tan solo 4 años.

Figura 4. Cantidad de fumigaciones por año en la hacienda Clementina en el 2008

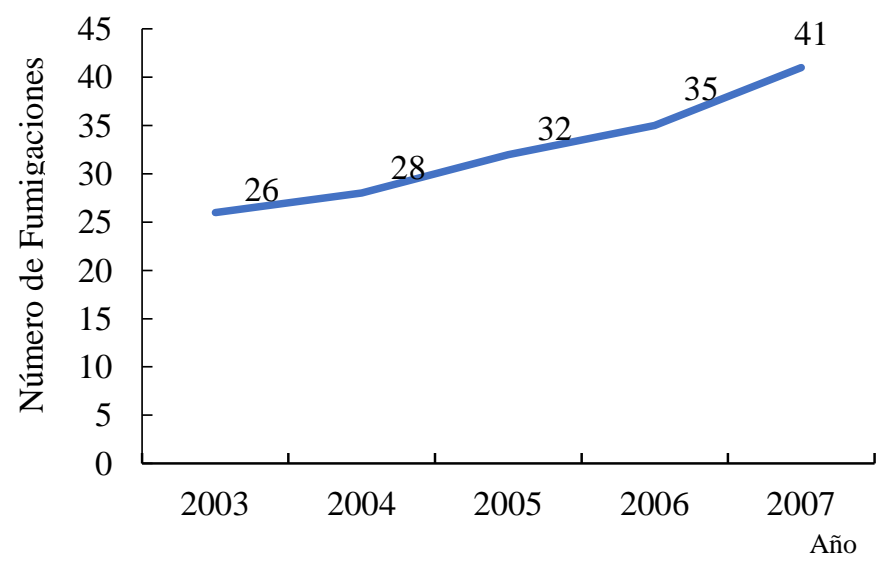

Fuente: Datos Hacienda Clementina

Riesgo para la producción sostenible de banano en Ecuador

Las enfermedades causadas por Mycosphaerella spp. colocan en riesgo algunos aspectos de la producción sostenible de banano considerando la definición de la agricultura sostenible que. "debe garantizar la seguridad alimentaria mundial y al mismo tiempo promover ecosistemas saludables y apoyar la gestión sostenible de la tierra, el agua y los recursos naturales" (FAO, 2015). Los problemas que genera la enfermedad aumentan el uso de agroquimicos que afectan la salud humana y del medio ambiente que interfiere en la posibilidad de que un sistema de producción sea conciente (FAO, 2015).

En el comercio entre Estados Unidos y otros paises se está basando en realizar acuerdos comerciales bilaterales, en donde se establece preferencias y medidas no solo económicas sino tambien la inclusión de reglamentaciones laborales y medioambientles, que permite una expansión del mercado mitigando los impactos socioambientales (OIT, 2007).

La tendencia de los mercados europeos se esta enfocando en obtener productos sostenibles, cabe mencionar que Holanda en el año 2015 exigía que el $20 \%$ de sus productos agrícolas provengan de plantaciones sostenibles y han establecido que a partir del 2020 el 100\% de los productos deberán ser sostenibles (HIDALGO, 2017).

Si el control de las manchas foliares de Sigatoka se basa en el uso continuo de fungicidas mas el aumento del número de fumigación por año, el aumento del costo de los agroinsumos que alcanzan el $15 \%$ y en otros casos el $30 \%$ del costo total de producción, costos que incrementan en función del tiempo como se muestra en el Figura 5. Siendo la enfermedad más nociva que actualmente se enfrentan las bananeras (PÉREZ et al., 2003). Considerando que la aparicion de esta enfermedad causó en la mayoria de paises productores de banano un tremendo impácto económico, solcial y afectación en la vialidad de plantaciones de los pequeños y medianos agricultores (STOVER, 1980).

Figura 5. Incremento entre 1995 y 2019 en el costo de producción del banano ecuatoriano de exportación

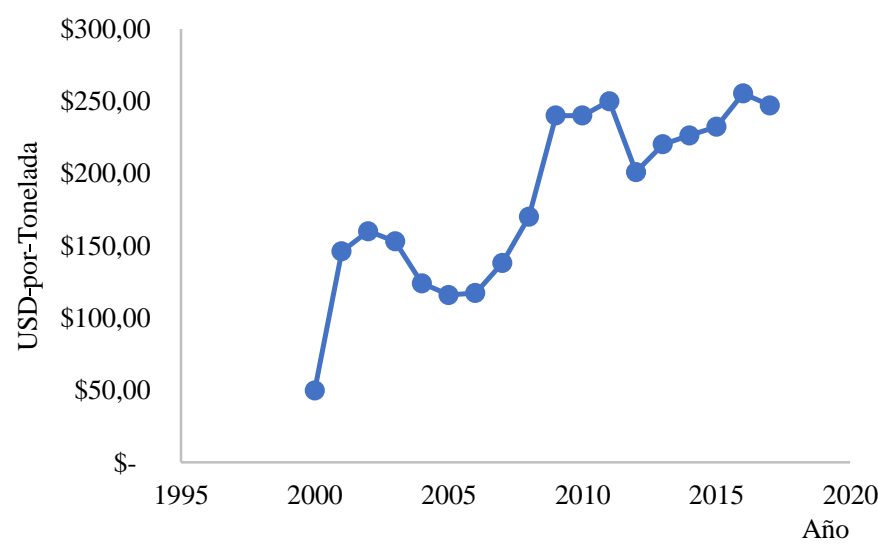

Fuente: FAOSTAT, 2019

Existen alternativas que podrían dar resultados en la producción sostenible de banano por mencionar una de ellas podría ser el control cultural como lo expresan Orozco, et al. (2008) que reduce las fuentes de inóculos del patógeno y las condiciones favorables para que se desarolle la enfermedad, es necesario conocer el comportamiento de la enfermedad y de las amenzas que con ella acarrea su mal manejo. 
Otra de las alternativas para lidiar con esta enfermedad es lograr una biodiversidad agrícola utilizando el mejoramiento genético de plantas, que tienen mayor probabilidades de ayudar al enfrentamiento de los retos en la sostenibilidad agrícola y contribuir a la seguridad alimentaria (NADAL et al., 2009).

\section{Discusión}

Existe en el Ecuador una disminución en la superficie plantada en un $31,71 \%$, se pierde un $2 \%$ de la superficie plantada anualmente debido a factores como la Sigatoka-negra una enfermedad foliar difícil de controlar. La forma de mitigar los daños causados por esta enfermedad es mediante fumigaciones de fungicidas de forma aérea, del $100 \%$ de las plantaciones bananeras el $66 \%$ se manejan mediante fumigaciones aéreas, lo cual, aumenta los costos de producción significativamente hasta en un 69,2\% en los últimos 17 años, lo cual es un riesgo para los pequeños productores que no pueden asumir estos costos.

La búsqueda de alternativas eficientes para controlar las manchas foliares de Sigatoka se debe de convertir en una prioridad para la investigación bananera mundial. Es un patógeno que representa una amenaza para los productores de banano. Ecuador que es un país bananero, siendo el mayor exportador a nivel mundial, debe fijar su mirada en el mejoramiento de los planes de control para la Sigatoka. Es poco probable que se logre una producción sostenible a largo plazo si no se buscan alternativas eficientes que puedan competir con el manejo convencional de la enfermedad y reducir los costos en el control.

La calidad del banano ecuatoriano es reconocida mundialmente, la amenaza que nos presenta esta enfermedad podría desprestigiar la calidad de la fruta producida en el Ecuador y complicaría el mercado para las exportaciones, otro problema a futuro es no poder llegar a producir banano en un sistema sostenible y como resultado de aquello, países que consumen nuestro producto se verían obligados a no permitir el ingreso de la fruta por políticas ya establecidas y colocaría en riesgo a toda la industria bananera en el Ecuador y cabe recalcar que es la industria agrícola más importante en los últimos años.

\section{CONCLUSIÓN}

El Ecuador al ser un país fuerte en producción de banano, si la enfermedad interfiere cada año sus exportaciones, afectaría a miles de familias que dependen de la estabilidad y de la productividad de estas plantaciones, dejaría de generar empleo a más de un millón de personas y colocaría en riesgo la seguridad alimentaria de miles de familias que dependen de este cultivo.

Los mercados internacionales y los consumidores se volverán cada vez más exigentes en la calidad de los productos sin desechar el cuidado del medio ambiente, en un futuro cercano los mercados de destino exigirán que los productos que ellos adquieran provengan de sistemas de producción sostenibles, lo cual sería un golpe más a la rentabilidad de las empresas bananeras ecuatorianas.

Las investigaciones en el manejo alternativo de esta enfermedad no son de carácter imprescindible para las asociaciones productoras o instituciones gubernamentales que buscan regular la exportación y comercialización de la fruta. La
Sigatoka-negra y amarilla tienen un antecedente de temer, este patógeno se vuelve cada vez más resistente a las aplicaciones de fungicidas y a largo plazo sería insostenible. Que se promueva el estudio de esta enfermedad y las posibilidades de reducir los impactos ambientales que acarrea el control actual, se convertiría en la mejor vía para el desarrollo sustentable de este cultivo y asegurar así más plazas de trabajo para los ecuatorianos y mayores generar más exportaciones.

Sería imposible llegar a un sistema de producción sostenible de banano en el Ecuador si no se adoptan medidas alternativas para el control de las manchas foliares por Sigatoka.

\section{REFERENCIAS}

ECUADOR. Banco Central. Reporte de Coyuntura sector Agropecuario. 4, 87, 84. 2015, Disponible: $<$ https://contenido.bce.fin.ec/documentos/PublicacionesNotas/C atalogo/Encuentas/Coyuntura/Integradas/etc201404.pdf>

Recuperado el 10/01/2019.

ECUADOR. Instituto Nacional de Estadísticas y Censos. Encuesta de superficie y producción agropecuaria continua. Banano, Ecuador. 2017.2 Disponible: $<$ https://www.ecuadorencifras.gob.ec/estadisticasagropecuarias-2/> Recuperado el 20/12/2018.

ECUADOR. Ministerio de Comercio Exterior. Informe sobre el sector bananero ecuatoriano. Quito. 2017. Disponible: $<$ https://www.proecuador.gob.ec/informe-de-banano-delecuador/> Recuperado el 20/12/2018.

ELBEHRI, A; CALBERTO, G.; STAVER, C.; HOSPIDO, A.; ROIBAS, L.; SKULLY, D.; SILES, P.; ARGUELLO, J.; BUSTAMANTE, I. SOTOMAYOR, A. Cambio climático y sostenibilidad del banano en el Ecuador: Evaluación de impacto y directrices de política. Roma: Organización de las naciones unidas para la alimentación y la agricultura (FAO). 2015. Disponible: 〈http://www.fao.org/3/a-i5116s.pdf> Recuperado el 21/01/2019.

FULLERTON, R; OLSEN, T. Pathogenic variability in Mycosphaerella fijensis Morelet, cause of black sigatoka in banana and plantain. New Zealand Journal of Crop and Horticulture Science, v.23, n.1, p39-48, 1995. $\underline{10.1080 / 01140671.1995 .9513866}$

GAUHL, F. Epidemiology and ecology of black sigatoka (Mycosphaerella fijensis Morelet) on plantain and banana (Musa spp.) in Costa Rica. Montpellier: INIBAP, 1994. 120p. Disponible: 〈https://hdl.handle.net/10568/101104〉 Recuperado el 14/02/2019.

GINEBRA Oficina Internacional del Trabajo. La promoción de empresas sostenibles. OIT, 2007. Disponible: <http://www.ilo.org/public/spanish/standards/relm/ilc/ilc96/pdf/ rep-vi.pdf> Recuperado el 10/08/2019.

GUZMÁN, M. Situación de la sigatoka negra en banano y plátano en el trópico americano. In: RIVAS, G.; ROSALES, F. (eds.) Manejo convencional y alternativo de sigatoka negra, 
nemátodos y otras plagas asociadas al cultivo de musáceas en los trópicos. Guayaquil: INIBAP, 2003. Cap. 1, p:11.

GUZMÁN, M; OROZCO SANTOS, M; PÉREZ, L. Las enfermedades sigatoka de las hojas del banano: dispersión, impacto y evolución de las estrategias de manejo en América Latina y el Caribe. In: XX Reunião Internacional da Associação para a Cooperação em Pesquisa e Desenvolvimento Integral das Musáceas (Bananas e Plátanos) Fortaleza. 2013.

HART, C. Doing a literature review, releasing the social science research imagination. London: SAGE publication, 2003. 250p.

HIDAGO, T. A. Estudio de concentración de pesticidas en aguas residuales de 10 fincas bananeras en las provincias de Los Ríos y Guayas, y su incidencia en los cuerpos de agua dulce. 2017. 94f. Defensa (Maestría en Ciencias Ambientales). Escuela Superior Politécnica Del Litoral, Guayaquil. 2017.

HIDALGO, M; TAPIA, A; RODRÍGUEZ, W; SERRANO, E. Efectos de la sigatoka negra (Mycosphaerella fijensis) sobre la fotosíntesis y transpiración foliar del banano (Musa sp. AAA, cv. Valery). Agronomía costarricense, v.30, n.1, p.35-41, 2006.

JONES, D. Handbook of diseases of banana, abaca and endset. Wallingford: CABI, 2000. 632p.

MARÍN, D.; ROMERO, R. El combate de la Sigatoka negra en divulgación científica al servicio del productor bananero nacional. Revista CORBANA. p104-129. 1998.

MARÍN, D.; ROMERO, R.; GUZMÁN, M.; SUTTON, T. B. Black sigatoka: an increasing threat to banana cultivation. Plant $\begin{array}{llll}\text { disease. } & \text { v.87, } & \text { n.3, } & \text { p208-222. }\end{array}$ 10.1094/PDIS.2003.87.3.208

MARTILLO, E. E.; SOLANO, P. Situación de la sigatoka negra en el Ecuador. In: RIVAS, G.; ROSALES, F. (eds.) Manejo convencional y alternativo de sigatoka negra, nemátodos y otras plagas asociadas al cultivo de musáceas en los trópicos. Guayaquil: INIBAP, 2003. Cap. 1, p:13.

MARTÍNEZ, E.; BARRIOS, G.; ROVESTI, L.; SANTOS, R. Manejo integrado de plagas. Manual. Habana: CNSV, 2006. 482p.

MEREDITH; D. S. Banana leaf spot disease (sigatoka) caused by Mycosphaerella musicola Leach. Kew. Commonwealth mycological institute, 1970. 147p.

MOURICHON, X.; FULLERTON, R. Geographical distribution of the two species Mycosphaerella musicola Leach (Cercospora musae) and M. fijiensis Morelet (C. fijiensis), respectively agents of sigatoka disease and black leaf streak disease in bananas and plantains. Fruits, v.45, n.3, p.213-218, 1992.

NADAL, R.; MANZO, G.; OROZCO, J.; GUZMÁN, N. Diversidad genética de bananos y plátanos (Musa spp.) determinada mediante marcadores RAPD. Revista Fitotecnia Mexicana. v.32, n.1, p1-7. 2009.
NÚÑEZ, R. El cultivo de banano. Programa nacional del banano. Ministerio de Agricultura Ganadería y Pesca. Quito. 1989.

Disponible:

<http://www.sica.gov.ec/agronegocios/biblioteca/Ing\%20Rizzo/ perf> Recuperado el 21/01/2019.

OROZCO, M.; OROZCO, J.; PÉREZ, O.; MANZO, G.; FARÍAS, J.; DA SILVA, W. Prácticas culturales para el manejo de la sigatoka negra en bananos y plátanos. Tropical Plant Pathology. v.33, n.3, p189-196. 2008. 10.1590/S1982$\underline{56762008000300003}$

PÉREZ, L.; ÁLVAREZ, J.; PÉREZ, M. Sigatoka negra causada por Mycosphaerella fijensis Morelet en Cuba: impacto económico, resistencia de los clones y manejo de la enfermedad. Fitosanidad, v.7, n.1, p31-41. 2003.

PÉREZ, L.; MAURI, F.; BARRANCO, B.; GARCÍA, G. Efficacy of EBI's fungicides in the control of Mycosphaerella fijensis Morelet on banana and plantains with treatments based on stage of evolution of the disease (biological warnings) in Cuba. In: Proceedings of the 6th international congress of plant pathology, 55. Montreal. 1993.

POLANCO, T.; CARLIER, J.; ZAPATER, M. Diagnóstico y distribución de Mycosphaerella spp. en musáceas de Republica Dominicana. Manejo Integrado de Plagas y Agroecología. San José. 2002. 64p.

SIMBIOTI-K. La Sigatoka amarilla del banano. 2016. Disponible: http://www.simbioti-k.com/la-sigatoka-amarilladel-banano/> Recuperado el 30 de 01 de 2019.

STOVER, R. Banana, plantain and abaca diseases. Kew. Commonwealth Myco-Logical Institute, 1972. 316p.

STOVER, R. Sigatoka leaf spot of bananas and plantains. Plant disease reporter. v.64, n.1, p750-756. 1980.

UNITED NATIONS. Food and Agricultural Organization (FAO). Agricultural data base. 2005. Disponible: <http://faostat.fao.org > Recuperado el 11/02/2019.

UNITED NATIONS. Food and Agricultural Organization. Enfermedades del banano y plátano en Venezuela. Medidas de control. 1999. Disponible: 〈http://www.ceniap.gov.ve/> Recuperado el 14/01/2019.

UNITED NATIONS. Food and Agricultural Organization. Objetivos de desarrollo sostenible. 2015. Disponible: $<$ http://www.fao.org/sustainable-development-

goals/overview/fao-and-post-2015/sustainable-agriculture/es/> Recuperado el 18/02/2019.

VERA, J. T. Evaluación del efecto sobre sigatoka negra, en hojas separadas de banano, Cavendish (variedad Williams), del extracto de Melaleuca alternifolia en 3 zonas del litoral ecuatoriano. 2013, 98f. Defensa (Ingeniero Agrícola y Biológico) Escuela Superior Politécnica Del Litoral, Guayaquil. 2013. 